\title{
Is VMAT Planning Feasible in Pediatric Neuroblastoma?
}

\author{
Anshuma Bansal ${ }^{1 *}$, Rakesh Kapoor ${ }^{1}$, Reena Kumari ${ }^{1}$, Amita Trehan $^{2}$ and KLN Rao ${ }^{3}$ \\ ${ }^{1}$ Department of Radiotherapy, Post Graduate Institute of Medical Education and Research, India \\ ${ }^{2}$ Department of Pediatric hematooncology, Chandigarh, India \\ ${ }^{3}$ Department of Pediatric Surgery, Post Graduate Institute of Medical Education and Research, India
}

Submission: June 28, 2017; Published: July 19, 2017

*Corresponding author: Anshuma Bansal, Assistant Professor, Department of Radiation Oncology, Post Graduate Institute of Medical Education and Research, Chandigarh, India, Email: dranshubansal3@gmail.com

\begin{abstract}
Aim: To do a dosimetric comparison between VMAT (Volumetric modulated arc radiotherapy) and 3DCRT (3-dimensional conformal radiotherapy) plans in pediatric abdominal neuroblastoma.

Methods: 15 patients with high risk abdominal neuroblastoma, treated between January 2012 and December 2015, with 3DCRT were reviewed. For comparison purpose, VMAT plans were also made on the planning CT scans. Volumetric and dosimetric changes in target volumes and normal structures were analyzed.

Results: VMAT plans covered target volumes more conformally than 3D-CRT (Conformity index: 1.12 vs 1.88; $\mathrm{p}=0.028$ ). The former was also superior to 3 DCRT plans in terms of lesser Dmean and V18 doses to ipsilateral kidney (Dmean: 15.45 Gy vs 18.27 Gy; p=0.02 and V18: $41.45 \%$ vs $60.65 \%$; $\mathrm{p}=0.004$ ), and contra lateral kidney (Dmean: 9.86 Gy vs $14.13 \mathrm{~Gy} ; \mathrm{p}=0.005$ and V18: 10.18 vs 34.39; $\mathrm{p}=0.002$ ), V12 and V15 parameters of liver (V12: $24.10 \%$ vs $31.89 \%$; $=0.000$ and V15: $14.03 \%$ vs $24.99 \%$; $p=0.001$ ) and Dmax spine (17.42 Gy vs $23.40 \mathrm{~Gy}$; $\mathrm{p}=0.000)$. The homogeneity index of VMAT plans was 0.01 compared to 0.87 with 3DCRT $(\mathrm{p}=0.02)$ Monitor units used to deliver radiation were 356 with VMAT compared to 246 only with 3DCRT $(\mathrm{p}=0.035)$. Time to deliver a single fraction with VMAT and 3D-CRT was 1.65 and $2.78 \mathrm{~min}$, respectively ( $\mathrm{p}=0.048$ ). The integral doses (Dmean) to non target tissues were however not significantly different between the two plans [4.97 Gy (VMAT) vs 4.07 Gy (3DCRT); $p=0.20]$.
\end{abstract}

Conclusion: The study demonstrates the superiority of VMAT plans over 3DCRT plans in terms of doses to organs at risk. The integral doses were also not significantly increased with VMAT planning, making these plans feasible to be used in pediatrics abdominal neuroblastoma.

Keywords: Neuroblastoma; Paediatrics; VMAT; 3DCRT

Abbreviations: CECT: Contrast enhanced computed tomography; 3DCRT: 3dimensional conformal radiotherapy; VMAT: Volumetric modulated arc radiotherapy; IMRT: Intensity modulated radiotherapy MLC: Multileaf collimator; GTV: The gross tumor volume; CTV: Clinical target volume; PTV: planning target volume; TPS: Treatment planning system

\section{Introduction}

Neuroblastomas account for $7-10 \%$ of all childhood cancers [1]. The most common site of primary tumor is retroperitoneum, ether in the adrenal gland or in paraspinal ganglions [1]. Role of local radiotherapy is well defined as a component of their management to help achieve local control [2]. Till date, 3dimensional conformal radiotherapy (3DCRT) is the standard conformal technique followed for such patients in majority of the institutes [2]. However, due to the location of target volumes near the critical structures like kidneys, spine, liver, and vertebral bodies, treating with 3D-CRT can be difficult.
The dose limiting toxicities can range from renal and hepatic toxicity to reduced growth of normal vertebral bodies causing scoliosis and kyphosis. VMAT (Volumetric modulated arc radiotherapy) is an advanced form of Intensity modulated radiotherapy (IMRT) that allows delivery of highly conformal radiotherapy in single or multiple gantry arc rotations by continuously modulating gantry speed, multileaf collimator (MLC) motion and dose rate, thereby significantly reducing time and monitor units (MU) required to deliver radiation $[3,4]$. The biggest advantage of VMAT is in its ability to treat target volumes wrapped around by critical structures, without exceeding the 
dose constraints for the latter [5]. Still, there is declination among the radiation oncologists to routinely implement IMRT planning in pediatric neuroblastomas due to the higher integral doses and risk of second malignancies [6].

The aim of this study was to do a dosimetric comparison between VMAT and 3DCRT plans in pediatric abdominal neuroblastoma to determine whether VMAT planning is feasible in such patients or not.

\section{Materials and Methods}

\section{Patients}

This is a retrospective study conducted in 15 patients of abdominal neuroblastoma registered in our department from January 2012 to December 2015. The patients were treated according to a modified COG protocol 3891 [7] for high risk neuroblastoma with 6 cycles of induction chemotherapy followed by surgical resection. This was followed by consolidation myelo-ablative chemotherapy, autologous stem cell transplant (ASCT), and localized abdominal radiotherapy. 3DCRT plans were generated to deliver radiotherapy, in accordance with the departmental protocol.

\section{Treatment planning}

All patients underwent planning contrast enhanced computed tomography (CECT) scans prior to treatment. The gross tumor volume (GTV) was contoured on the axial slices based on the preoperative post-chemotherapy CT scan, and the planning CT. An additional $10 \mathrm{~mm}$ margin was added to generate the clinical target volume (CTV). This margin was then edited to include only $2 \mathrm{~mm}$ of the kidneys and the liver. Finally, the CTV was expanded by $10 \mathrm{~mm}$ margin cranio-caudally, and by $3 \mathrm{~mm}$ in the lateral and antero-posterior directions to generate the planning target volume (PTV). The target dose ranged from 21.8 Gy for completely resected tumors, while higher doses (up to $25.8 \mathrm{~Gy}$ ) were prescribed for gross residual disease. Treatment planning was performed on treatment planning system (TPS). Both the 3DCRT plans and the VMAT plans were made for comparison purpose and the final dose distribution was calculated.

\section{DCRT planning}

The plan was made by using 2-3 angled photon fields using MLC in order to spare normal structures as much as possible. All the plans were aimed to deliver the $95 \%$ isodose volume to the PTV.

\section{VMAT planning}

The plans were aimed to deliver the $95 \%$ isodose volume to the PTV, while respecting the dose constraints to the organs at risk. For the OAR, the primary objective criteria entailed more than $80 \%$ of at least one kidney received less that $18 \mathrm{~Gy}$, and less than $50 \%$ of the liver volume received more than 8 Gy [V8 Gy], and the volume of the liver receiving more than 15 Gy [V15 Gy] was less than $25 \%$. The vertebral body was marked as a secondary target volume aiming at delivering as much homogeneous dose as possible within the bony tissue of the adjacent vertebrae with a minimum dose of $15 \mathrm{~Gy}$ and a maximum dose as low as possible so as to avoid potential asymmetric skeletal growth in the future. Treatment was delivered using 3DCRT plans on Linear accelerator using $6 \mathrm{MV}$ energy.

\section{Dosimetric comparison}

For PTV coverage: Comparison was made between the PTV coverage among the two plans by 3DCRT and VMAT in terms of V95 and V100 (Volume receiving 95\% and 100\% of the prescribed dose respectively), conformity and homogeneity indexes, time and monitor units used to complete the treatment, and the integral doses among the two plans. For normal tissues: Comparisons were made for the doses received by bilateral kidneys, ipsilateral kidney, contra lateral kidney, liver and spinal cord by the 3DCRT and VMAT plans.

\section{Statistical analysis}

SPSS v. 19.0 (SPSS Inc., Chicago, IL) software was used for statistical analysis. Wilcoxan signed-rank test was used to compare dosimetric parameters between the 3DCRT and VMAT plans. Paired T-test was used to compare the means. The statistical difference was considered significant at $\mathrm{p}<0.05$.

\section{Results}

\section{Patient and Tumor profile}

Table 1: Demographic profile.

\begin{tabular}{|c|c|}
\hline Characteristic & $\mathbf{N}=\mathbf{1 5}$ \\
\hline Age (years) [mean \pm range] & {$[4.8 \pm 2]$} \\
\hline Gender & 12 \\
\hline Male & 3 \\
\hline Female & \\
\hline Laterality & 3 \\
\hline Right & 11 \\
\hline Left & \\
\hline
\end{tabular}

Table 2: Tumor and organ at risk profile.

\begin{tabular}{|c|c|c|c|}
\hline \multirow{2}{*}{ Parameter } & \multicolumn{3}{|c|}{ Volume (cc) } \\
\hline & Mean (SE) & Mean (SE) & Mean (SE) \\
\hline PTV & $\begin{array}{c}227.68 \\
( \pm 18.12)\end{array}$ & $225.6( \pm 70.19)$ & $\begin{array}{c}106.70- \\
318.33\end{array}$ \\
\hline $\begin{array}{c}\text { Bilateral } \\
\text { Kidneys }\end{array}$ & $87.64( \pm 3.63)$ & $88.80( \pm 14.06)$ & $65.40-110.10$ \\
\hline $\begin{array}{c}\text { Ipsilateral } \\
\text { Kidney }\end{array}$ & $45.01( \pm 2.50)$ & $44.70( \pm 9.70)$ & $27.80-60.80$ \\
\hline $\begin{array}{c}\text { Contralateral } \\
\text { Kidney }\end{array}$ & $42.60( \pm 2.09)$ & $42.30( \pm 8.13)$ & $23.40-55.10$ \\
\hline Liver & $\begin{array}{c}457.26 \\
( \pm 15.28)\end{array}$ & $452.8( \pm 59.19)$ & $\begin{array}{c}318.80- \\
541.90\end{array}$ \\
\hline
\end{tabular}

SE: Standard error of mean; SD: Standard deviation. 


\section{Cancer therapy \& Oncology International Journal}

At the time of starting radiotherapy the mean age of the patients was $4.8 \pm 2$ years. There were 12 males and 3 females. All patients were treated for high risk disease (Table 1). The radiation dose ranged from 21 to 25.5 Gy with a mean dose of $24.06 \pm 1.16$, using a daily fraction 1.5-1.8 Gy. The PTV volume ranged from 106.70 to 318.33 cc with a median volume of
225.6 cc. The median volume of liver was $452.8( \pm 59.19)$ cc and for bilateral kidneys was $88.80( \pm 14.06)$ cc (Table 2). VMAT compared to 3DCRT (Tables $3 \& 4$ ) shows the dosimetric comparison of VMAT versus 3DCRT plans. PTV dosimetry (Table 3).

Table 3: Dose variation between 3DCRT and VMAT plans (PTV dosimetry).

\begin{tabular}{|c|c|c|c|}
\hline \multirow{2}{*}{ Dose parameters } & $\begin{array}{c}\text { 3DCRT } \\
\text { mean } \pm \text { SE }\end{array}$ & $\begin{array}{c}\text { VMAT } \\
\text { mean } \pm \text { SE }\end{array}$ \\
\hline \multicolumn{2}{|c|}{ PTV } \\
\hline V100 (\%) & $96.65 \pm 2.37$ & $98.72 \pm 1.60$ & 0.38 \\
\hline V95 (\%) & $99.17 \pm 0.94$ & $100.02 \pm 0.17$ & 0.04 \\
\hline Dmax (Gy) & $25.52 \pm 0.52$ & $26.68 \pm 0.43$ & 0.23 \\
\hline Dmin (Gy) & $21.27 \pm 0.57$ & $20.46 \pm 0.34$ & 0.19 \\
\hline Conformality indexa & $1.88 \pm 0.12$ & $0.12 \pm 0.06$ & 0.028 \\
\hline Homogenity & $0.10 \pm 0.06$ & & 0.05 \\
\hline indexb & & $356 \pm 23$ & 0.035 \\
\hline Monitor units & $246 \pm 42$ & $1.65 \pm 0.03$ & 0.046 \\
\hline
\end{tabular}

Dmean: mean dose; PTV: planning target volume; SE: standard error of mean.

V100 and V95: Volume receiving $95 \%$ and $100 \%$ of the prescribed dose respectively.

Conformality indexa: V95/PTV volume (Ideal value $=1$ ).

Homogenity index ${ }^{\mathrm{b}}$ : D2- D98/D $\times$ $\times 100($ Ideal value $=0$ ).

PTV coverage was adequately achieved by both the plans. The Dmax and Dmin doses were also not different among the two plans. VMAT plans covered target volumes more conformally than 3DCRT (Conformity index: 1.12 vs 1.88 ; $\mathrm{p}=0.028$ ). The homogeneity index of VMAT plans was 0.01 compared to 0.87 with 3DCRT $(p=0.02)$. Monitor units used to deliver radiation

were 356 with VMAT compared to 246 only with 3DCRT ( $p=$ 0.035). The main advantage of VMAT plans over 3DCRT plans was reduced treatment time and faster treatment delivery $(1.65$ and $2.78 \mathrm{~min}$, respectively $(\mathrm{p}=0.048)$ ), which shortened the anesthesia duration, since most of the patients were treated under anesthesia.

\section{OAR dosimetry (Table 4)}

Table 4: Dose variation between 3DCRT and VMAT plans (OAR dosimetry).

\begin{tabular}{|c|c|c|c|}
\hline Dose parameters & 3DCRT mean $\pm \mathrm{SE}$ & VMAT mean \pm SE & p-value \\
\hline \multicolumn{4}{|c|}{ Ipsilateral kidney } \\
\hline Dmean (Gy) & $18.27 \pm 1.12$ & $15.45 \pm 1.09$ & 0.02 \\
\hline V18 (\%) & $60.65 \pm 7.08$ & $41.45 \pm 5.96$ & 0.004 \\
\hline \multicolumn{4}{|c|}{ Contra lateral kidney } \\
\hline Dmean (Gy) & $14.13 \pm 1.57$ & $9.86 \pm 0.91$ & 0.005 \\
\hline V18 (\%) & $34.39 \pm 8.09$ & $10.18 \pm 3.54$ & 0.002 \\
\hline \multicolumn{4}{|c|}{ Bilateral kidneys } \\
\hline Dmean (Gy) & $16.16 \pm 0.88$ & $12.67 \pm 3.28$ & 0.000 \\
\hline V18 (\%) & $47.29 \pm 4.75$ & $26.00 \pm 4.27$ & 0.000 \\
\hline \multicolumn{4}{|c|}{ Liver } \\
\hline Dmean (Gy) & $8.59 \pm 1.10$ & $7.36 \pm 0.84$ & 0.07 \\
\hline V8 (\%) & $41.40 \pm 6.00$ & $42.08 \pm 5.97$ & 0.75 \\
\hline V12 (\%) & $31.89 \pm 4.95$ & $24.10 \pm 4.70$ & 0.000 \\
\hline V15 (\%) & $24.99 \pm 4.90$ & $14.03 \pm 3.05$ & 0.001 \\
\hline
\end{tabular}




\begin{tabular}{|c|c|c|c|}
\hline \multicolumn{5}{|c|}{ Spinal cord } \\
\hline Dmax (Gy) & $23.40 \pm 0.56$ & $17.42 \pm 0.48$ & 0.000 \\
\hline
\end{tabular}

Dmean: mean dose; SE: standard error of mean.

V8, V12, V15 and V18: Volume of organ at risk (OAR) receiving 8 Gy, 12 Gy, 15 Gy and 18 Gy respectively.

VMAT plans however were dosimetrically superior to 3DCRT plans in terms of doses received by ipsilateral kidbey (Dmean and V18), contra lateral Kidney (Dmean and V18), liver (V12 and V15) and spinal cord (Dmax). For ipsilateral kidney, Dmean was $15.45 \pm 1.09$ Gy by VMAT plans versus $18.27 \pm 1.12$ Gy by 3DCRT plans ( $p=0.02$ ), and V18 was $41.45 \pm 5.96 \%$ by VMAT plans versus $60.65 \pm 7.08 \%$ by 3DCRT plans ( $p=0.01)$. This shows that VMAT plans can achieve $19.2 \%$ reduction in volume of ipsilateral kidney receiving doses more than 18 Gy. Dmean and V18 for contra lateral kidney was 4.27 Gy and $24.21 \%$ less respectively for VMAT plans compared to 3DCRT plans. Similarly for bilateral kidneys, Dmean and V18 was 3.49 Gy and $21.29 \%$ less respectively for VMAT plans compared to 3DCRT plans.
For liver, while the mean doses and V8 were not statistically different for VMAT and 3DCRT plans, there was definite disparity among V12 and V15 parameters, which denote threshold for venoocclusive disease in pediatric patients. V12 was $24.10 \pm$ $4.70 \%$ by VMAT plans versus $31.89 \pm 4.95 \%$ by 3DCRT plans ( $\mathrm{p}=0.000$ ), and V15 was $14.03 \pm 3.05 \%$ by VMAT plans versus $24.99 \pm 4.90 \%$ by 3DCRT plans $(\mathrm{p}=0.001)$. This denotes that VMAT plans can reduce chances of venoocclusive disease in pediatric patients compared to patients treated with 3DCRT plans. While the maximum tolerance limit for spinal cord was achieved by both the plans, still the spinal cord Dmax was 5.98 Gy less with VMAT plans versus 3DCRT plans, which was statistically significant $(\mathrm{p}=0.000)$.

\section{Integral doses (Table 5)}

Table 5: Dose variation between 3DCRT and VMAT plans [Non tumor tissue (NTT) dosimetry].

\begin{tabular}{|c|c|c|c|}
\hline Dose parameters & 3DCRT mean \pm SE & VMAT bv & p-value \\
\hline NTT Volume (cc) & $4422.20 \pm 266.14$ & $25.96 \pm 0.44$ & 0.13 \\
\hline Dmax (Gy) & $25.52 \pm 0.50$ & $0.007 \pm 0.002$ & 0.28 \\
\hline Dmin (Gy) & $0.03 \pm 0.006$ & $4.07 \pm 0.21$ & 0.20 \\
\hline
\end{tabular}

NTT Volume: Body volume - Total PTV volume.

The volume of normal tissues receiving low dose radiation [body minus PTV] was similar among 3DCRT and VMAT. The integral doses (Dmean) to these non target tissues were not significantly different between the two plans [4.97 Gy (VMAT) vs 4.07 Gy (3DCRT); $\mathrm{p}=0.20]$.

\section{Discussion}

The results of our study clearly indicate the superiority of VMAT plans compared to 3DCRT in terms of high conformality and homogeneity. Also, the VMAT plans demonstrated lesser Dmean and V18 doses to ipsilateral kidney, contra lateral kidney, V12 and V15 parameters of liver and Dmax spine doses. Time to deliver a single fraction with VMAT was also significantly less compared to 3DCRT. The highlight of our study is that it revealed no significant difference in the integral doses (Dmean) to non target tissues among 3DCRT and VMAT plans.

In the literature, most of the studies done in tumors other than pediatric abdominal tumors, (gliomas, pelvic tumors, head and neck malignancies) prove VMAT plannings to be superior to 3DCRT plannings, in terms of conformality for target coverage and tendency to save critical structures [3,4,8-10]. Limited data is available for VMAT radiotherapy planning in pediatric abdominal tumors, due to the concern for large low dose volume regions. Majority of such children planned with VMAT had special indications like reirradiation, single kidney, horseshoe kidneys, where dose constraint to normal tissues was anyway difficult to achieve with 3DCRT.

Shaffer et al. [11] compared RapidArc, fixed field IMRT (CIMRT), 3D-CRT, and a parallel-opposed pair (POP) among eight children of retroperitoneal tumors. The study found that RapidArc and cIMRT covered target volumes more conformally than 3D-CRT and POP (P=0.012). There was no difference in the ability to meet kidney dose constraints. A significantly lower volume of the liver received 12Gy with cIMRT or RapidArc compared with 3D-CRT $(\mathrm{P}=0.028)$. Time to deliver a single fraction with RapidArc, POP, 3D-CRT, and cIMRT was $1.25 \pm$ $0.01,1.38 \pm 0.10,2.6 \pm 0.45$, and $4.02 \pm 1.12 \mathrm{~min}$, respectively $(\mathrm{P}=0.012)$. Monitor units for a single fraction with POP, 3D-CRT, RapidArc, and cIMRT were $203 \pm 26,235 \pm 32,325 \pm 71$, and $665 \pm 215$, respectively $(\mathrm{P}<0.05)$. The study concluded that RapidArc and cIMRT were generally no better dosimetrically than conformal techniques. 3D-CRT was more conformal but had higher treatment time. RapidArc resulted in a major reduction in time but had required MUs to deliver the radiation. The results were quite similar to the present study.

Chojnacka [12] treated a $3 \cdot 5$-year-old patient with Wilms tumour of the right kidney after the preoperative chemotherapy and the right nephrectomy during which the tumour rupture 
was found. The child was eligible for whole peritoneal cavity irradiation with VMAT using double arc, to the dose of $19 \cdot 5$ Gy in 13 fractions. The main constraint of this therapy was the left kidney which received the mean dose of 12 Gy in the both plans. The 3DCRT plan was unsatisfactory due to worse target volume covering. The PTV95\% amounted 88\% for 3DCRT versus $99 \%$ for VMAT. Again homogeneity and conformity index were best achieved with VMAT planning.

Hence we see that in pediatric abdominal tumors, the results of VMAT are superior to 3DCRT in terms of conformality, homogenity and normal tissue sparing. Despite this, there are certain factors which limit the routine implementation of IMRT planning in pediatric retroperitoneal neuroblastomas. One of the most important factors is the exposure of a larger volume of normal tissues to low doses of radiotherapy i.e. high integral doses received by non target tissues, also referred to as low dose volume regions, which potentially increases the risk of secondary malignancy. The integral dose equals to the mean dose multiplied by the volume of non target tissues. The low dose volume is defined as the volume receiving a total dose of 2 Gy or more [13]. This low dose is primarily caused by a leakage through the accelerator head, jaws and MLCs together with the internal scatter within the patient. Children are a major concern as they have more proliferating tissues with more numbers of stem cells compared to the adults. It is estimated that the incidence of secondary malignancies could almost double with IMRT techniques compared with conventional techniques (from $1 \%$ to $1.75 \%$ for patients surviving 10 years) [14].

The theoretical risk of secondary malignancy induction with VMAT is generally considered lower, compared to conventional fixed field IMRT, because VMAT uses fewer MU, which in turn leads to lower integral doses. However the focus of this study was to determine, whether the integral doses with VMAT plans are higher, lower or comparable to 3DCRT plans, as 3DCRT technique is still the standard treatment technique followed for pediatric abdominal tumors till date. Also, the literature has been reviewed to determine the same. Cabrera et al. [15], treated seven pediatric neuroblastoma patients with VMAT, while simultaneously evaluating dose distribution to normal tissues proximal and distal to PTV by VMAT as compared to 3D-CRT and IMRT. Two regions were defined for estimation of dose distribution to normal tissues. The first region covered the entire body except for the PTV and the lower extremities.

The second region was a $5-\mathrm{cm}$ ring around the PTV. Dmax, Dmean, and integral dose for both regions in the three plans were analyzed. The study found that Dmax values were similar in all three techniques. Dmean values were similar between IMRT and VMAT ( $\mathrm{p}=0.043)$ and both lower than in 3D-CRT $(\mathrm{p}=$ 0.018). There were significant differences in MUs with the lowest mean value found in the VMAT plan. Integral dose was higher for 3DCRT ( $p=0.018)$, and similar between IMRT and VMAT ( $p$
$=0.043$ ). The study concluded that the incidence of secondary cancers may be lower in VMAT-treated patients, as compared to IMRT-treated patients. Nevertheless, these conclusions should be considered with caution, given the small sample size. S Patel [16] compared VMAT, helical tomotherapy (HT) and 3D-CRT for craniospinal irradiation (CSI) in five children with medulloblastoma.

The mean nontarget tissue integral dose for VMAT of 80.8 J was significantly lower than for 3D-CRT (91.5 J, P = 0.04) and HT (95.6 J, P = 0.04). Body and nontarget tissue integral doses were lowest with VMAT in every patient. The study concluded that VMAT provides comparable normal tissue sparing with tomotherapy and may reduce the integral dose. These findings have potential implications in the risk of the development of late adverse effects and radiation-related second malignancies in children with curable primary disease. In our study also, the integral doses (Dmean) received by non target tissues were not significantly different between the VMAT and 3DCRT plans [4.97 Gy (VMAT) vs 4.07 Gy (3DCRT); $p=0.20$ ].

Though most of the studies in literature correlate integral doses with the risk of secondary malignancy, still the reliability of most of the secondary cancer risk models is an issue. Marco et al. [17] treated eight patients with intrathoracic lesions with stereotactic body radiation therapy (SBRT), 23 Gy $\times 1$ fraction. All patients were then replanned for 3D-CRT, maintaining the same target coverage and applying a dose scheme of 2 Gy $\times$ 32 fractions. Total integral dose received by non-tumor tissue (NTID) for both techniques was calculated, along with estimating possible correlations between NTID and radiation-induced secondary malignancy risk using a mechanistic model. The study found that despite the fact that for all patients integral dose is higher for SBRT treatments than 3D-CRT ( $p=0.002)$, secondary cancer risk associated to SBRT patients is significantly smaller than that calculated for 3D-CRT $(p=0.001)$. The study concluded that integral dose may not be a good estimator for quantifying cancer induction.

From the above discussion, it is evident that VMAT planning is feasible in pediatric neuroblastomas and can be routinely implemented in practice. However, the development of reliable secondary cancer risk models and correlating these with the integral doses seems to be a key issue in fractionated radiotherapy.

\section{Conclusion}

VMAT has the clear superiority over the conventional conformal methods with regard to the improvement in the dose conformity and normal tissue sparing. There is the evidence to show that VMAT has a definite place in the paediatric radiotherapy. However, a longer follow-up will be required to actually quantify the risk of late toxicity. 


\section{Ethical Statement}

The study is only a dosimetric comparison of radiotherapy plans, on already treated patients of neuroblastoma. Therefore, permissions from pertinent body were not sought.

\section{References}

1. De Grazia E, Cimador M, De Bernardi B (1997) Recent advances on retroperitoneal neuroblastoma. Arch Ital Urol Androl 69: 233-240.

2. Haas-Kogan DA, Swift PS, Selch M, Haase GM, Seeger RC, et al. (2003) Impact of radiotherapy for high-risk neuroblastoma: a children's cancer group study. Int J Radiat Oncol Biol Phys 56(1): 28-39.

3. Clivio A, Fogliata A, Franzetti-Pellanda A, Nicolini G, Vanetti E, et al. (2009) Volumetric modulated arc radiotherapy for carcinomas of the anal canal: A treatment planning comparison with fixed field IMRT. Radiother Oncol 92(1): 118-124.

4. Cozzi L, Dinshaw KA, Shrivastava SK, Mahantshetty U, Engineer $\mathrm{R}$, et al. (2008) A treatment planning study comparing volumetric arc modulation with RapidArc and fixed field IMRT for cervix uteri radiotherapy. Radiother Oncol 89(2): 180-191.

5. Otto K (2008) Volumetric modulated arc therapy: IMRT in a single gantry arc. Med Phys 35(1): 310-317.

6. D’Arienzo M, Masciullo SG, Sanctis V, Osti MF, Chiacchiararelli L, et al. (2012) Integral Dose and Radiation-Induced Secondary Malignancies: Comparison between Stereotactic Body Radiation Therapy and ThreeDimensional Conformal Radiotherapy. Int J Environ Res Public Health 9(11): 4223-4240.

7. Matthay KK, Reynolds CP, Seeger RC, Shimada H, Adkins ES, et al. (2009) "Long-term results for children with high-risk neuroblastoma treated on a randomized trial of myeloablative therapy followed by 13cis-retinoic acid: a children's oncology group study". J Clin Oncol 27(7): 1007-1013.

8. Beltran C, Gray J, Merchant T (2012) Intensity-modulated arc therapy for pediatric posterior fossa tumors. Int J Radiat Oncol Biol Phys 82(2): 299-304.
9. Wagner D, Christiansen H, Wolff H, Vorwerk H (2009) Radiotherapy of malignant gliomas: comparison of volumetric single arc technique (RapidArc), dynamic intensity-modulated technique and 3D conformal technique. Radiother Oncol 93(3): 593-596.

10. Shaffer R, Nichol AM, Vollans E, Fong M, Nakano S, et al. (2010) A comparison of volumetric modulated arc therapy and conventional intensity modulated radiotherapy for frontal and temporal highgrade gliomas. Int J Radiat Oncol Biol Phys 76(4): 1177-1184.

11. Shaffer R, Vollans E, Vellani R, Welsh M, Moiseenko V, (2011) A radiotherapy planning study of RapidArc, IMRT, 3-D conformal radiotherapy and parallel opposed beams in the treatment of pediatric retroperineal tumors. Pediatr Blood Cancer 56(1): 16-23.

12. Chojnacka M, Zygmuntowicz-Piętka A, Semaniak A, Pędziwiatr K Dąbrowski R, et al. (2015) Volumetric modulated arc therapy versus 3D conformal radiotherapy for selected childhood neoplasms. Journal of Radiotherapy in Practice 14: 35-42.

13. Zaghloul MS (2013) Intensity modulated radiotherapy (IMRT) for pediatric cancer patients: The advantage and fear of second malignant neoplasm. Journal of the Egyptian National Cancer Institute 25(1): 1-3.

14. Hall EJ, Wuu CS (2003) Radiation-induced second cancers: the impact of 3D-CRT and IMRT. Int J Radiat Oncol Biol Phys 56: 83-88.

15. Cabrera P, Pérez JM, Peinado J, Ruiz Y, Rubio M, et al. (2013) Integral dose in the treatment of pediatric neuroblatoma with 3DCRT-IMRTVMAT. Reports of Practical Oncology \& Radiotherapy 18: S296.

16. Patel S, Drodge S, Jacques A, Warkentin H, Powell K, et al. (2015) A Comparative Planning Analysis and Integral Dose of Volumetric Modulated Arc Therapy, Helical Tomotherapy, and Three-dimensional Conformal Craniospinal Irradiation for Pediatric Medulloblastoma. Journal of Medical Imaging and Radiation Sciences 46(2):134-140.

17. D'Arienzo M, Masciullo SG, Sanctis VD, Osti MF, Chiacchiararelli L, et al (2012) Integral dose and radiation-induced secondary malignancies: comparison between stereotactic body radiation therapy and three-dimensional conformal radiotherapy. International journal of environmental research and public health 9(11): 4223-4240.

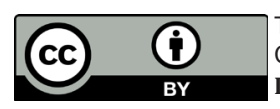

This work is licensed under Creative Commons Attribution 4.0 License DOI: 10.19080/CTOIJ.2017.06.555680

\section{Your next submission with Juniper Publishers} will reach you the below assets

- Quality Editorial service

- Swift Peer Review

- Reprints availability

- E-prints Service

- Manuscript Podcast for convenient understanding

- Global attainment for your research

- Manuscript accessibility in different formats

( Pdf, E-pub, Full Text, Audio)

- Unceasing customer service

Track the below URL for one-step submission https://juniperpublishers.com/online-submission.php 